20. Miller, J. R. \& Moon, R. T. Analysis of the signalling activities of localization mutants of beta-catenin during axis specification in Xenopus. J. Cell Biol. 139, 229-243 (1997).

21. Weitzel, H. E. \& Ettensohn, C. A. beta-catenin is differentially degraded along the animal-vegetal axis of early sea urchin embryos in a GSK-3b-dependent manner. Dev. Biol. 247, 479a (2002).

22. Yost, C. et al. The axis-inducing activity, stability, and subcellular distribution of beta-catenin is regulated in Xenopus embryos by glycogen synthase kinase 3. Genes Dev. 10, 1443-1454 (1996).

23. Ding, Y. \& Dale, T. Wnt signal transduction: kinase cogs in a nano-machine? Trends Biochem. Sci. 27, 327-329 (2002).

24. Heasman, J. et al. Overexpression of cadherins and underexpression of beta-catenin inhibit dorsal mesoderm induction in early Xenopus embryos. Cell 79, 791-803 (1994).

25. Montross, W. T., Ji, H. \& McCrea, P. D. A beta-catenin/engrailed chimera selectively suppresses Wnt signalling. J. Cell Sci. 113, 1759-1770 (2000).

26. Freeman, G. \& Miller, R. L. Hydrozoan eggs can only be fertilized at the site of polar body formation. Dev. Biol. 94, 142-152 (1982).

27. Larabell, C. A. et al. Establishment of the dorso-ventral axis in Xenopus embryos is presaged by early asymmetries in beta-catenin that are modulated by the Wnt signalling pathway. J. Cell Biol. 136, 1123-1136 (1997)

28. Mao, C.-A. et al. Altering cell fates in sea urchin embryos by overexpressing SpOtx, an orthodenticlerelated protein. Development 122, 1489-1498 (1996).

29. Klymkowsky, M. W. \& Hanken, J. Whole-mount staining of Xenopus and other vertebrates. Methods Cell Biol. 36, 419-441 (1991).

30. McCrea, P. D., Brieher, W. M. \& Gumbiner, B. M. Induction of a secondary body axis in Xenopus by antibodies to beta-catenin. J. Cell Biol. 123, 477-484 (1993).

Supplementary Information accompanies the paper on www.nature.com/nature.

Acknowledgements We thank Y. Marikawa, B. Klein and members of our laboratories for critical reading of the manuscript and for suggestions and P. McCrea for the gift of affinity-purified $\beta$-catenin antibody. This work was supported by grants from the NSF and the Hawaii Community Foundation to A.H.W. and by grants from the NSF and NASA to M.Q.M.

Competing interests statement The authors declare that they have no competing financial interests.

Correspondence and requests for materials should be addressed to A.H.W. (athula@hawaii.edu) or M.Q.M. (mqmartin@hawaii.edu).

\section{Angiotensin-converting enzyme 2}

is a functional receptor for the SARS coronavirus

Wenhui Li ${ }^{1}$, Michael J. Moore ${ }^{1}$, Natalya Vasilieva ${ }^{2}$, Jianhua Sui ${ }^{3}$, Swee Kee Wong ${ }^{1}$, Michael A. Berne ${ }^{4}$, Mohan Somasundaran ${ }^{5}$, John L. Sullivan ${ }^{5}$, Katherine Luzuriaga ${ }^{5}$, Thomas C. Greenough ${ }^{5}$, Hyeryun $\mathrm{Choe}^{2}$ \& Michael Farzan

${ }^{1}$ Partners AIDS Research Center, Brigham and Women's Hospital, Department of Medicine (Microbiology and Molecular Genetics), ${ }^{2}$ Perlmutter Laboratory,

Pulmonary Division, Children's Hospital, Department of Pediatrics,

${ }^{3}$ Dana-Farber Cancer Institute, Department of Medicine, Harvard Medical School, Boston, Massachusetts 02115, USA

${ }^{4}$ Tufts University Core Facility, Tufts University School of Medicine, Boston, Massachusetts 02111, USA

${ }^{5}$ Program in Molecular Medicine, University of Massachusetts Medical School, Worcester, Massachusetts 01605, USA

Spike (S) proteins of coronaviruses, including the coronavirus that causes severe acute respiratory syndrome (SARS), associate with cellular receptors to mediate infection of their target cells ${ }^{1,2}$. Here we identify a metallopeptidase, angiotensin-converting enzyme 2 (ACE2) $)^{3,4}$, isolated from SARS coronavirus (SARS$\mathrm{CoV}$ )-permissive Vero E6 cells, that efficiently binds the $\mathrm{S} 1$ domain of the SARS-CoV $S$ protein. We found that a soluble form of ACE2, but not of the related enzyme ACE1, blocked association of the S1 domain with Vero E6 cells. 293T cells transfected with ACE2, but not those transfected with human immunodeficiency virus-1 receptors, formed multinucleated syncytia with cells expressing $S$ protein. Furthermore, SARS-CoV replicated efficiently on ACE2-transfected but not mock-transfected 293T cells. Finally, anti-ACE2 but not antiACE1 antibody blocked viral replication on Vero E6 cells. Together our data indicate that ACE2 is a functional receptor for SARS-CoV.

So far two types of coronavirus surface receptor have been identified $^{5}$. The group II coronavirus mouse hepatitis virus (MHV) uses murine carcinoembryonic antigen-related cell adhesion molecules (CEACAMs), members of the immunoglobulin superfamily of receptors ${ }^{6}$. A number of group I coronaviruses, for example human coronavirus 229E, transmissible gastroenteritis virus and feline infectious peritonitis virus, require the zinc metalloprotease aminopeptidase N (APN, CD13) for entry into their target cells $^{7-9}$. Recently, a distinct coronavirus has been identified as the aetiological agent of SARS, an acute pulmonary syndrome characterized by an atypical pneumonia that results in progressive respiratory failure and death in close to $10 \%$ of cases ${ }^{10-13}$. Analysis of the SARS-CoV genome suggests that this virus does not belong to any of the three defined coronavirus groups, and that the SARS-CoV S protein is similarly distinct ${ }^{14,15}$. Similar to the analogous human immunodeficiency virus (HIV) and influenza proteins, the $S$ proteins of some coronaviruses-including MHV and the group III coronavirus infectious bronchitis virus-are cleaved into two subunits ( 1 1 and $\mathrm{S} 2$ ) by a cellular protease in virus-producing cells ${ }^{16,17}$. The $S$ proteins of other coronaviruses, including those of group I and probably SARS-CoV, are not cleaved in virus-producing cells ${ }^{14,15,18}$. Nonetheless, S1 and S2 domains of these latter S proteins can be identified through their homology with the S1 and S2 subunits of cleaved coronavirus S proteins. The S1 domains of all characterized coronaviruses mediate an initial high-affinity association with their respective receptors ${ }^{19-21}$.

The African green monkey kidney cell line Vero E6 permits replication of SARS-CoV ${ }^{10}$. We first investigated whether the S1 domain of the SARS-CoV S protein could bind to Vero E6 cells. Figure 1a demonstrates that a protein expressing residues 12-672 of the SARS-CoV S protein, fused to the Fc domain of human immunoglobulin- $\gamma 1$ (IgG1; S1-Ig), specifically recognized a moiety present on Vero E6 cells but did not bind human kidney 293T cells. Using this same fusion protein (Fig. 1c) or a carboxy terminally tagged form of the S1 domain (Fig. 1b), a protein band of approximately $110 \mathrm{kDa}$ could be immunoprecipitated from metabolically labelled Vero E6 cells lysed with $0.3 \% n$-decyl- $\beta$-Dmaltopyranoside in phosphate-buffered saline. When the immunoprecipitated protein was incubated with PNGase F, an enzyme that removes $N$-glycosylation, two bands were observed at approximately $80-85$ and $100 \mathrm{kDa}$ (Fig. 1c, lanes 3 and 4).

We then analysed the $110 \mathrm{kDa}$ band by trypsin digestion and mass spectrometry. Three human proteins were identified whose sequences were consistent with the masses of tryptic fragments obtained from this band. Two of these proteins, myosin $1 \mathrm{~b}$ and major vault protein, do not localize to the cell surface and are ubiquitously expressed, therefore we did not analyse them further. Eight independent tryptic fragments consistent with sequences comprising $17 \%$ of the amino acid sequence of human ACE2 were also identified (Supplementary Information). Because both the tissue distribution and subcellular localization of ACE2 are appropriate for a receptor for SARS-CoV $3,22,23$, we cloned it from complementary DNA obtained from human lung for further analysis.

293T cells transfected with plasmid expressing ACE2, but not those transfected with vector alone, were specifically recognized by S1-Ig (Fig. 2a). A soluble form of ACE2, but not that of the related enzyme ACE1, blocked the association of S1-Ig with Vero E6 cells (Fig. 2b). Furthermore, S1-Ig immunoprecipitated a soluble form of ACE2 (Fig. 2c, lane 5) that was recognized by an anti-ACE2 antibody (lane 8 ). When the approximately $110 \mathrm{kDa}$ soluble form of ACE2 was incubated with PNGase F, an $85 \mathrm{kDa}$ band was observed (Fig. 2c, lanes 5 and 6), indicating that the lower band observed in 
PNGase-F-treated Vero E6 immunoprecipitates is ACE2 (Fig. 1c, lanes 3 and 4). These data demonstrate a specific, high-affinity association between the S1 domain of the SARS-CoV S protein and ACE2.

Proteins that mediate fusion between viral and cellular membranes can in some cases also do so between cells that express the viral fusion protein and those that express the viral receptor. For instance, cells expressing the HIV-1 envelope glycoprotein gp160 can form multinucleated syncytia with cells expressing the HIV-1 receptors CD4 and CCR5 (ref. 24). Because syncytia have been observed in Vero E6 cultures infected with SARS-CoV, in SARS$\mathrm{CoV}$-infected primates, and in SARS patients ${ }^{10,12,13}$, we investigated whether 293T cells expressing the SARS-CoV S protein could fuse with 293T cells expressing ACE2. Figure 3a shows that these cells formed syncytia efficiently. As expected, 293T cells transfected with CD4 and CCR5 formed many syncytia with cells expressing HIV-1 gp160 (top left), but not with cells expressing SARS-CoV S protein (bottom left). In contrast, 293T cells expressing ACE2 did not form syncytia with cells expressing gp160 (top right), but
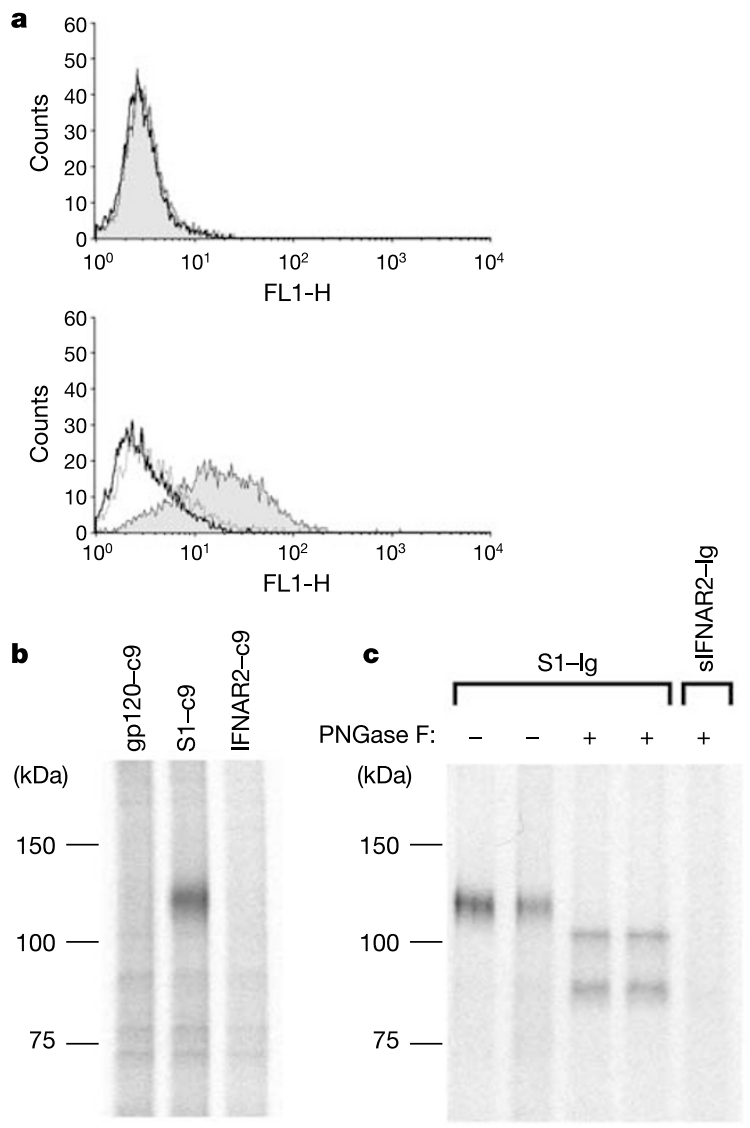

Figure $1 \mathrm{~A} 110 \mathrm{kDa}$ protein associates with the S1 domain of SARS-CoV S protein. a, A fusion protein of the $S 1$ domain ( $S 1-\mathrm{lg}$, shaded area), or of the first 327 residues of that domain (S1(327)-Ig, dotted line) with the Fc domain of human IgG1, or culture medium alone (thick line) was incubated with 293T (top panel) or Vero E6 (bottom panel) cells. Binding of fusion proteins to cells was measured by flow cytometry using a FITC-labelled anti-human IgG secondary antibody. b. Metabolically labelled Vero E6 cell lysates were immunoprecipitated with HIV-1 gp120, SARS-CoV S1 domain, or the ectodomain of human IFNAR2, each containing a tag (C9) at its $\mathrm{C}$ terminus, and an anti-tag antibody. Immunoprecipitates were analysed by SDS-PAGE. c, Labelled Vero E6 cell lysates were immunoprecipitated with S1-Ig or soluble IFNAR2-Ig.

Immunoprecipitates were treated or not, as indicated, with PNGase F, and analysed by SDS-PAGE. formed many large syncytia with cells expressing $\mathrm{S}$ protein (bottom right). 293T cells expressing $\mathrm{S}$ protein also efficiently formed syncytia with ACE2- but not mock-transfected HeLa cells (not shown). Syncytia formation between S protein- and ACE2-expressing cells was blocked by more than $95 \%$ by affinity-purified antibody raised against ACE2, but not by control antibody (Fig. 3b). These data demonstrate that the ability of the SARS$\mathrm{CoV} S$ protein to mediate cell-cell fusion is dependent on the presence of ACE2.

We then investigated the ability of ACE2 to mediate viral replication. ACE2- and mock-transfected 293T cells were incubated in the presence or absence of SARS-CoV. After 2 days in culture, numerous detached, round and floating cells were observed in cultures of infected ACE2-transfected cells (not shown), consistent with viral replication. In contrast, infected mock-transfected cells and uninfected ACE2-transfected cells were indistinguishable from uninfected control cells, and these cells displayed none of the signs of cytopathicity that were observed in infected ACE2-expressing cells. Figure 4 shows that ACE2- but not mock-transfected 293T cells supported efficient replication of SARS-CoV. We measured viral genomic RNA in cell supernatants by semi-quantitative polymerase chain reaction with reverse transcription (RT-PCR). We found that viral genome copies in ACE2-transfected cells increased by more than 100,000-fold in the first $48 \mathrm{~h}$ (Fig. 4a, bottom panel).
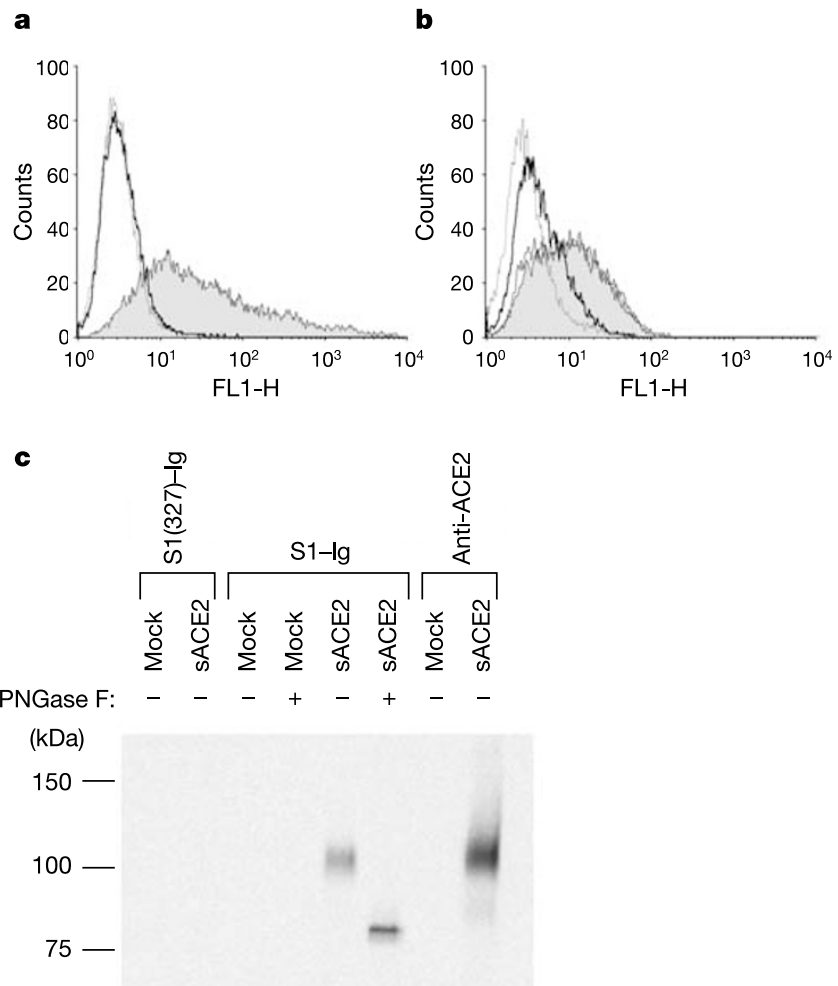

Figure 2 A high-affinity association between ACE2 and the S1 domain. a, 293T cells transfected with plasmid encoding ACE2 (shaded area and dotted line), or with vector alone (thick solid line), were analysed by flow cytometry with S1-Ig (shaded area and thick solid line) or S1(327)-Ig (dotted line). b, Vero E6 cells were incubated with S1-Ig (shaded area, thin solid line and thick solid line), or with medium alone (dotted line), in the presence of soluble ACE1 (thin solid line) or ACE2 (thick solid line), or without either protein (shaded area and dotted line). c, Supernatants of radiolabelled 293T cells transfected with plasmid encoding soluble ACE2 or with vector alone (mock) were immunoprecipitated with S1(327)-Ig, S1-Ig, or an anti-ACE2 antibody. Immunoprecipitates were treated or not, as indicated, with PNGase F, and analysed by SDS-PAGE. 
In contrast, genome copies in mock-transfected cells increased tenfold in the same period (top panel), indicating some basal replication on $293 \mathrm{~T}$ cells. In addition, virus titres in supernatants from infected ACE2- and mock-transfected cells were measured by titration onto Vero E6 cells. As shown in Fig. 4b, virus stock obtained $96 \mathrm{~h}$ after infection from ACE2-transfected cells efficiently replicated on Vero E6 cells, with visible cytopathic effect (CPE) up to and including the highest dilution assayed $(1: 6,561)$, whereas virus stock obtained from mock-transfected cells induced no CPE beyond a 1:27 dilution. Consistent with the basal replication observed in both infection assays, minor expression of ACE2 on 293T cells was subsequently detected by an anti-ACE2 antibody (Supplementary Information). We also investigated the ability of anti-ACE2 antibody to inhibit viral replication on Vero E6 cells. Anti-ACE1 antibody had no observable effect on virally induced cytopathicity, whereas anti-ACE2 antibody inhibited cytopathicity in a dosedependent manner (Fig. 4c). Collectively, these data show that ACE2 contributes substantially to the efficiency of SARS-CoV replication. a HIV- 1 gp $160+$ CD $4 / C C R 5$

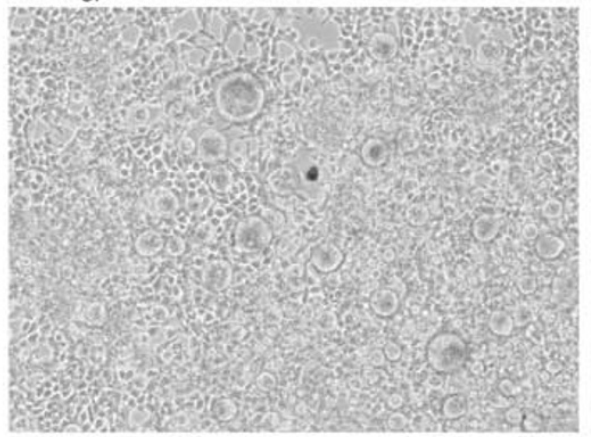

S protein + CD $4 / C C R 5$

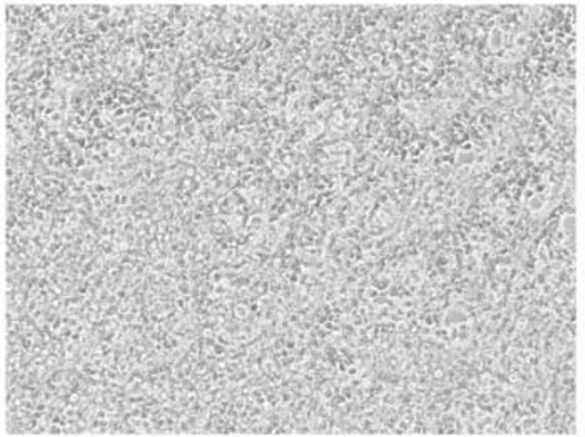

b S protein + ACE2 + control Ab

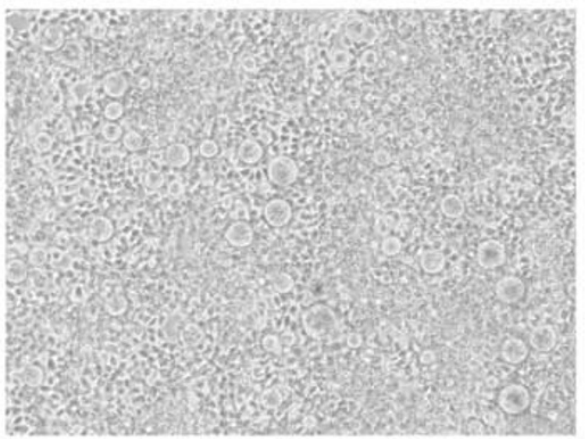

We show here that ACE2 can be immunoprecipitated from Vero E6 cells by the S1 domain of the SARS-CoV S protein, that it mediates fusion with S-protein-expressing cells, and that it promotes viral replication in a cell line otherwise inefficient for SARS-CoV infection. Moreover, syncytia formation and viral replication were specifically inhibited by an anti-ACE2 antibody. These data demonstrate that ACE2 is a functional receptor for SARS-CoV. Real-time PCR analysis of 72 human tissues has demonstrated efficient expression of ACE2 messenger RNA in the bronchus and lung parenchyma as well as in the heart, kidney and gastrointestinal tract $^{23}$. Lung and kidney are also the primary sites of expression of murine ACE2 (ref. 22). This tissue distribution is consistent with the pathology of SARS, which is characterized by an acute infection of the lungs ${ }^{10-12}$. SARS-CoV has also been found in kidney tissue of infected patients ${ }^{10}$, and it replicates efficiently on the primate kidney cell lines FRhK-4 and Vero E6 (refs 10, 12). Active viral replication in the small and large intestine has been observed ${ }^{25}$. Detection of virus in the faeces ${ }^{11}$ may reflect expression of ACE2 message in these tissues $^{23}$. The physiological role of ACE2 in most of these tissues has

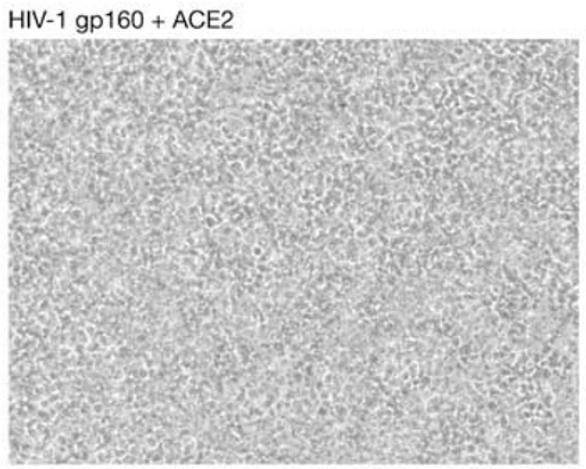

S protein + ACE2

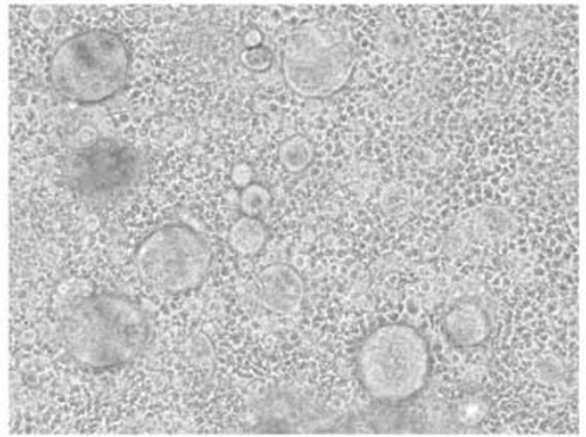

$\mathrm{S}$ protein + ACE2 + anti-ACE2 Ab

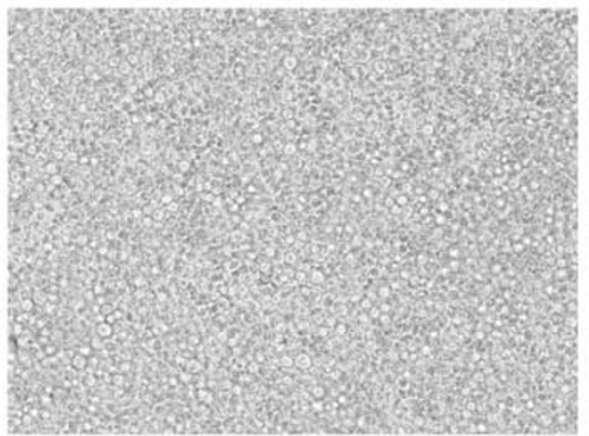

Figure 3 Syncytia formation between S-protein- and ACE2-expressing cells. a, 293T cells transfected with plasmids encoding the HIV-1 envelope glycoprotein gp160 (top row) or SARS-CoV S protein (bottom row) were mixed at a 1:1 ratio with 293T cells transfected with plasmids encoding HIV-1 receptors CD4 and CCR5 (left column) or ACE2 (right column). b. 293T cells transfected with ACE2 plasmid were mixed at a 1:1 ratio with 293T cells transfected with plasmid encoding S protein, in the presence of $10 \mu \mathrm{g} \mathrm{ml}^{-1}$ goat polyclonal control antibody (left) or goat polyclonal anti-ACE2 antibody (right). Ab, antibody. 
not been defined, although ACE2 is thought to be an essential regulator of cardiac function ${ }^{26}$. Candidate substrates, some of which regulate vasopermeability, have been identified ${ }^{27}$. It is unclear whether SARS-CoV interferes with ACE2 enzymatic activity in a manner that contributes to the pathogenesis of SARS.

CD13, the receptor for a number of coronaviruses, is, like ACE2, a zinc metalloprotease; however, apart from this commonality the two receptors are widely divergent. This may suggest that some property of this class of proteases contributes to viral replication. However, mutations in the catalytic site of CD13 do not interfere with tissue-culture replication of transmissible gastroenteritis virus,

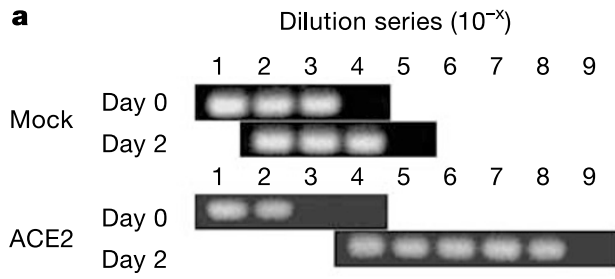

b
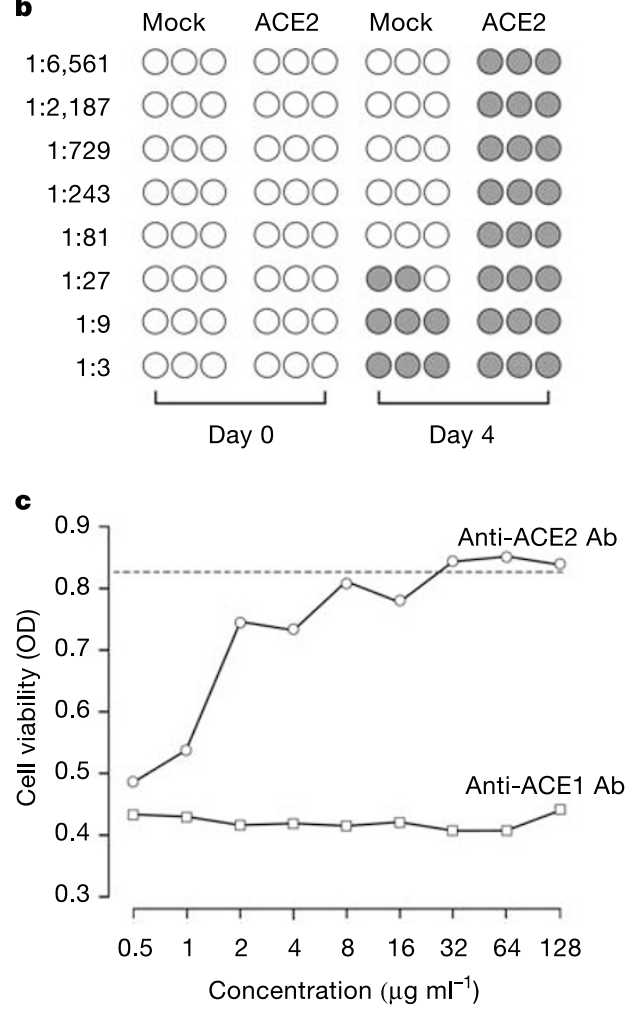

Figure 4 Efficient replication of SARS-CoV in the presence of ACE2. a, Mock- or ACE2-transfected 293T cells were infected with SARS-CoV for $1 \mathrm{~h}$, and cell supernatants sampled 0 and $48 \mathrm{~h}$ after washing. Viral RNA was measured by RT-PCR at varying dilutions of supernatant. The endpoint of dilution is shown for each group. $\mathbf{b}$, Supernatants (collected at days 0 and 4) of infected mock- and ACE2-transfected 293T cells were incubated at the indicated dilutions in triplicate with Vero E6 cells plated on 96-well microtitre plates. Cytopathic effect, indicated by a shaded circle, was monitored for each well 3 days after Vero E6 cell infection. c, Duplicate samples of Vero E6 cells were incubated with affinity-purified goat anti-ACE1 or anti-ACE2 antibody, at the indicated concentrations, before infection with SARS-CoV. Cells were washed and cell viability, shown as the average optical density (OD) at $490 \mathrm{~nm}$ wavelength, was assayed using CellTiter 96 . The dotted line indicates viability of uninfected cells. No toxicity was observed in uninfected cells treated with the maximum concentration of either antibody. which uses this enzyme as a receptor ${ }^{7,28}$. Similarly, our preliminary studies showed that syncytia formation mediated by SARS-CoV S protein remained efficient when two active-site histidines of ACE2 were modified to asparagine (Supplementary Information).

A number of antibodies, peptides and small compounds have been shown to bind to ACE2 (refs 29, 30). It is possible that some of these may be useful in the treatment of SARS, either by blocking the S-protein-binding site, or by inducing a conformation in ACE2 that is unfavourable to binding or fusion. Alternatively, a soluble form of the receptor itself may slow viral replication in an infected individual. Identification of ACE2 as a SARS-CoV receptor will facilitate description of the receptor-binding domain of the $S$ protein, presumably the most effective target epitope of an S1-proteinbased subunit vaccine. Also, it is likely that a cell line approved for vaccine production and made permissive for viral replication by ACE2 expression will be the most efficient large-scale producer of a whole-killed or attenuated virus for use as a vaccine. A mouse transgenic for human ACE2 may be useful as an animal model of SARS. Finally, study of the interaction between the SARS-CoV S protein and ACE2 of other animals may provide insights into the origins of the virus. Thus, if SARS returns as a threat to human health, these studies may contribute to its control.

\section{Methods}

\section{Immunoprecipitation and identification of ACE2}

Vero E6 cells were metabolically labelled for $48 \mathrm{~h}$ with $\left[{ }^{35} \mathrm{~S}\right]$ cysteine and $\left[{ }^{35} \mathrm{~S}\right]$ methionine, and lysed in $1.5 \mathrm{ml}$ per $100-\mathrm{mm}$ dish of $0.3 \% n$-decyl- $\beta$-D-maltopyranoside (DDM, Anatrace) in phosphate-buffered saline (PBS) containing protease-inhibitor cocktails (Sigma and Roche) and $0.2 \mathrm{mM}$ phenylmethylsulphonyl fluoride (Sigma). After removal of cell debris by centrifugation, lysate was incubated for $1 \mathrm{~h}$ at room temperature with $2.5 \mu \mathrm{g}$ purified S1-Ig or human interferon- $\alpha$ receptor 2 (IFNAR2)-Ig fusion constructs and protein A Sepharose. Alternatively, C-terminally C9-tagged forms of the S1 domain (S1-C9) or of control proteins (HIV-1 gp120-C9 and IFNAR2-C9) were incubated with the antibody 1D4 (National Cell Culture Center) together with protein A Sepharose. Precipitates were washed twice in 0.3\% DDM/PBS and once in PBS alone. Bound proteins were eluted in reducing Laemmli sample buffer at $55^{\circ} \mathrm{C}$ or in non-reducing buffer at $37^{\circ} \mathrm{C}$ for $10 \mathrm{~min}$. Proteins were separated by SDS-polyacrylamide gel electrophoresis (PAGE) on $8 \%$ Tris-glycine gels (Invitrogen). Using this approach approximately $5 \times 10^{7}$ unlabelled Vero E6 cells were used to generate a distinct band of $110 \mathrm{kDa}$ that could be readily visualized by Coomassie staining. This band was excised from the gel and incubated with trypsin, and the masses of tryptic fragments were determined by matrix-assisted laser desorption ionization time-of-flight mass spectrometry. Masses were compared with possible tryptic fragments of proteins in the GenBank database using Sequest software.

\section{Receptor expression measurement on Vero E6 and 293T cells}

Plasmids encoding the signal sequence of CD5 and a fusion of the S1 domain of the SARS-CoV S protein (residues 12-672), or the first 316 residues of that domain (12-327), with the Fc region of human IgG1 (S1-Ig and S1(327)-Ig, respectively) were transfected into $293 \mathrm{~T}$ cells, and immunoglobulin fusion proteins were purified on protein A Sepharose beads. A total of $5 \times 10^{5} 293 \mathrm{~T}$ cells transfected with ACE2-expressing or control plasmids, or the same number of untransfected Vero E6 cells, were incubated with $15 \mu \mathrm{g}$ $\mathrm{ml}^{-1}$ of S1-Ig or S1(327)-Ig in a volume of $100 \mu \mathrm{l}$. In some cases, $15 \mu \mathrm{g} \mathrm{ml}^{-1}$ of soluble forms of ACE1 or ACE2 (R\&D Systems) was also included. Cells were washed in PBS with $0.5 \% \mathrm{BSA}$ and $0.1 \% \mathrm{NaN}_{3}$, incubated with FITC-labelled goat anti-human IgG Fc (Sigma), and analysed by flow cytometry.

\section{Syncytia formation}

$293 \mathrm{~T}$ cells, approximately $50 \%$ confluent on a six-well plate, were transfected by the calcium-phosphate method with plasmids encoding a codon-optimized form of the SARS-CoV S protein or of the HIV-1 envelope glycoprotein gp160 (ADA isolate). In parallel, 293T or HeLa cells were transfected with plasmids encoding ACE2 or the HIV-1 receptors CD4 and CCR5. One day after transfection, cells were trypsinized and those expressing viral-envelope proteins were mixed with cells expressing receptors at a 1:1 ratio, and plated on 12- or 24-well plates. At 24-48 h after mixing, multinucleated giant cells were observed. In some cases $10 \mu \mathrm{g} \mathrm{ml}^{-1}$ of goat anti-ACE2 antibody or of goat anti-ACE1 antibody (R\&D Systems) was added at the time of cell mixing.

\section{Infection of mock- and ACE2-transfected 293T cells}

SARS-CoV (Urbani strain), provided by L. Anderson (Centers for Disease Control and Prevention), was passaged on Vero E6 cells. 293T cells transfected in $25 \mathrm{~cm}^{2}$ flasks with pcDNA3.1 alone or pcDNA3.1 expressing ACE2 were infected for $1 \mathrm{~h}$ with $1.4 \times 10^{3}$ $\mathrm{TCID}_{50}(50 \%$ tissue-culture infectious dose) of SARS-CoV, as measured by endpoint titration on Vero E6 cells, or left uninfected, and washed twice in culture medium. Cells were monitored for cytopathic effect for 4 days. Aliquots of cell supernatants were collected 0,48 and $96 \mathrm{~h}$ after wash. RNA was recovered using a viral RNA mini-prep kit 
(Qiagen). Semi-quantitative RT-PCR was performed using a nested protocol as described by ref. 11. Positive control RNA was provided by C. Drosten (Bernhard Nocht Institute for Tropical Medicine, National Reference Center for Tropical Diseases). Virus titration was performed by seeding $5 \times 10^{3}$ Vero E6 cells per well in 96-well microtitre plates 1 day before infection. Culture supernatant from infected $293 \mathrm{~T}$ cells was added to the first set of wells in triplicate and serially diluted. Cells were monitored for CPE 3 days after infection of Vero E6 cells. The effect of affinity-purified goat anti-ACE1 or -ACE2 antibody on SARS-CoV-induced cytopathicity was measured by reading absorbance at $492 \mathrm{~nm}$ of cells incubated with CellTiter 96 (Promega)

Received 14 September; accepted 23 October 2003; doi:10.1038/nature02145.

Gallagher, T. M. \& Buchmeier, M. J. Coronavirus spike proteins in viral entry and pathogenesis. Virology 279, 371-374 (2001)

Holmes, K. V. SARS-associated coronavirus. N. Engl. J. Med. 348, 1948-1951 (2003).

3. Donoghue, M. et al. A novel angiotensin-converting enzyme-related carboxypeptidase (ACE2) converts angiotensin I to angiotensin 1-9. Circ. Res. 87, E1-E9 (2000).

4. Tipnis, S. R. et al. A human homolog of angiotensin-converting enzyme. Cloning and functional expression as a captopril-insensitive carboxypeptidase. J. Biol. Chem. 275, 33238-33243 (2000).

5. Holmes, K. V. et al. Coronavirus receptor specificity. Adv. Exp. Med. Biol. 342, 261-266 (1993).

6. Dveksler, G. S. et al. Several members of the mouse carcinoembryonic antigen-related glycoprotein family are functional receptors for the coronavirus mouse hepatitis virus-A59. J. Virol. 67, 1-8 (1993).

Delmas, B. et al. Aminopeptidase $\mathrm{N}$ is a major receptor for the entero-pathogenic coronavirus TGEV. Nature 357, 417-420 (1992).

8. Tresnan, D. B. \& Holmes, K. V. Feline aminopeptidase $\mathrm{N}$ is a receptor for all group I coronaviruses Adv. Exp. Med. Biol. 440, 69-75 (1998).

9. Yeager, C. L. et al. Human aminopeptidase $\mathrm{N}$ is a receptor for human coronavirus 229E. Nature 357, $420-422$ (1992).

10. Ksiazek, T. G. et al. A novel coronavirus associated with severe acute respiratory syndrome. N. Engl. J. Med. 348, 1953-1966 (2003).

11. Drosten, C. et al. Identification of a novel coronavirus in patients with severe acute respiratory syndrome. N. Engl. J. Med. 348, 1967-1976 (2003)

12. Kuiken, T. et al. Newly discovered coronavirus as the primary cause of severe acute respiratory syndrome. Lancet 362, 263-270 (2003)

13. Fouchier, R. A. et al. Aetiology: Koch's postulates fulfilled for SARS virus. Nature 423, 240 (2003).

14. Rota, P. A. et al. Characterization of a novel coronavirus associated with severe acute respiratory syndrome. Science 300, 1394-1399 (2003)

15. Marra, M. A. et al. The Genome sequence of the SARS-associated coronavirus. Science 300, 1399-1404 (2003).

16. Sturman, L. S. \& Holmes, K. V. Proteolytic cleavage of peplomeric glycoprotein E2 of MHV yields two 90K subunits and activates cell fusion. Adv. Exp. Med. Biol. 173, 25-35 (1984).

17. Jackwood, M. W. et al. Spike glycoprotein cleavage recognition site analysis of infectious bronchitis virus. Avian Dis. 45, 366-372 (2001)

18. Spaan, W., Cavanagh, D. \& Horzinek, M. C. Coronaviruses: structure and genome expression. J. Gen Virol. 69, 2939-2952 (1988)

19. Bonavia, A., Zelus, B. D., Wentworth, D. E., Talbot, P. J. \& Holmes, K. V. Identification of a receptor-binding domain of the spike glycoprotein of human coronavirus HCoV-229E. J. Virol. 77, 2530-2538 (2003)

20. Breslin, J. J. et al. Human coronavirus 229E: receptor binding domain and neutralization by soluble receptor at 37 degrees C. J. Virol. 77, 4435-4438 (2003).

21. Kubo, H., Yamada, Y. K. \& Taguchi, F. Localization of neutralizing epitopes and the receptor-binding site within the amino-terminal 330 amino acids of the murine coronavirus spike protein. J. Virol. 68 , 5403-5410 (1994).

22. Komatsu, T. et al. Molecular cloning, mRNA expression and chromosomal localization of mouse angiotensin-converting enzyme-related carboxypeptidase (mACE2). DNA Seq. 13, 217-220 (2002).

23. Harmer, D., Gilbert, M., Borman, R. \& Clark, K. L. Quantitative mRNA expression profiling of ACE 2, a novel homologue of angiotensin converting enzyme. FEBS Lett. 532, 107-110 (2002).

24. Choe, H. et al. The beta-chemokine receptors CCR 3 and CCR 5 facilitate infection by primary HIV-1 isolates. Cell 85, 1135-1148 (1996).

25. Leung, W. K. et al. Enteric involvement of severe acute respiratory syndrome-associated coronaviru infection. Gastroenterology 125, 1011-1017 (2003).

26. Crackower, M. A. et al. Angiotensin-converting enzyme 2 is an essential regulator of heart function. Nature 417, 822-828 (2002).

27. Vickers, C. et al. Hydrolysis of biological peptides by human angiotensin-converting enzyme-related carboxypeptidase. J. Biol. Chem. 277, 14838-14843 (2002).

28. Delmas, B. et al. Determinants essential for the transmissible gastroenteritis virus-receptor interaction reside within a domain of aminopeptidase-N that is distinct from the enzymatic site. J. Virol. 68 , 5216-5224 (1994).

29. Huang, L. et al. Novel peptide inhibitors of angiotensin-converting enzyme 2. J. Biol. Chem. 278, 15532-15540 (2003)

30. Dales, N. A. et al. Substrate-based design of the first class of angiotensin-converting enzyme-related carboxypeptidase (ACE2) inhibitors. J. Am. Chem. Soc. 124, 11852-11853 (2002).

Supplementary Information accompanies the paper on www.nature.com/nature.

Acknowledgements We thank J. Coderre for assistance with RT-PCR, M. Kirk for editing, and S. H. Wang, E. Kieff, J. Sodroski, C. Gerard and N. Gerard for guidance and helpful conversations

Competing interests statement The authors declare that they have no competing financial interests.

Correspondence and requests for materials should be addressed to H.C (hyeryun.choe@tch.harvard.edu) or M.F. (farzan@mbcrr.harvard.edu).

\section{Altered thymic T-cell selection due to a mutation of the ZAP-70 gene causes autoimmune arthritis in mice}

\author{
Noriko Sakaguchi ${ }^{1,2 *}$, Takeshi Takahashi ${ }^{1 \star}$, Hiroshi Hata ${ }^{1}$ \\ Takashi Nomura ${ }^{1}$, Tomoyuki Tagami ${ }^{1}$, Sayuri Yamazaki ${ }^{1}$, \\ Toshiko Sakihama ${ }^{1}$, Takaji Matsutani ${ }^{3}$, Izumi Negishi ${ }^{4}$, \\ Syuichi Nakatsuru ${ }^{1}$ \& Shimon Sakaguchi ${ }^{1,2}$
}

${ }^{1}$ Department of Experimental Pathology, Institute for Frontier Medical Sciences, Kyoto University, Kyoto 606-8507, Japan

${ }^{2}$ Laboratory for Immunopathology, RIKEN Research Center for Allergy and Immunology, Yokohama 230-0045, Japan

${ }^{3}$ Department of Immunology, Shionogi Institute for Medical Science, Settsu, Osaka 566-0022, Japan

${ }^{4}$ Department of Dermatology, Gunma University Hospital, Maebashi, Gunma 371-8511, Japan

* These authors contributed equally to this work

Rheumatoid arthritis (RA), which afflicts about $1 \%$ of the world population, is a chronic systemic inflammatory disease of unknown aetiology that primarily affects the synovial membranes of multiple joints ${ }^{1-3}$. Although $\mathrm{CD}^{+} \mathrm{T}$ cells seem to be the prime mediators of RA, it remains unclear how arthritogenic $\mathrm{CD}^{+} \mathrm{T}$ cells are generated and activated ${ }^{1-3}$. Given that highly self-reactive T-cell clones are deleted during normal T-cell development in the thymus, abnormality in T-cell selection has been suspected as one cause of autoimmune disease ${ }^{4,5}$. Here we show that a spontaneous point mutation of the gene encoding an $\mathrm{SH} 2$ domain of ZAP-70, a key signal transduction molecule in $\mathrm{T}$ cells ${ }^{6}$, causes chronic autoimmune arthritis in mice that resembles human RA in many aspects. Altered signal transduction from T-cell antigen receptor through the aberrant ZAP-70 changes the thresholds of $T$ cells to thymic selection, leading to the positive selection of otherwise negatively selected autoimmune $T$ cells. Thymic production of arthritogenic $T$ cells due to a genetically determined selection shift of the T-cell repertoire towards high self-reactivity might also be crucial to the development of disease in a subset of patients with RA.

The SKG strain, which spontaneously develops chronic arthritis, is derived from our closed breeding colony of $\mathrm{BALB} / \mathrm{c}$ mice. Joint swelling with hyperaemia became macroscopically evident in SKG mice at about 2 months of age, initially at a few interphalangeal joints of the forepaws, then progressing in a symmetrical fashion to swelling of other finger joints of the forepaws and hindpaws, and larger joints (wrists and ankles) (Fig. 1a-d). Knee, elbow, shoulder or vertebral joints were rarely affected except for the joint at the base of the tail in aged SKG mice (Fig. 1e). Radiographic examination revealed destruction and fusion of the subchondral bones, joint dislocation, and osteoporosis by $8-12$ months of age (Fig. 1f-i). Despite suffering from such severe chronic arthritis, most SKG mice survived well to 1 year of age, generally with more severe arthritides in females (Fig. 1j).

Histology of the swollen joints showed severe synovitis with massive subsynovial infiltration of neutrophils, lymphocytes, macrophages and plasma cells, villus proliferation of synoviocytes accompanying pannus formation and neovascularization, and neutrophil-rich exudates in the joint cavity (Fig. 1k, 1), with progression of synoviocyte proliferation, pannus-eroded adjacent cartilage and subchondral bone. Immunohistochemical staining revealed that $\mathrm{CD} 4^{+} \mathrm{T}$ cells predominantly infiltrated the subsynovial tissue (Fig. 1m, n). As extra-articular manifestations of the disease, most (more than 90\%) mice older than 6 months of age developed interstitial pneumonitis with various degrees of perivascular and 